\title{
A mathematical model using routine serum biomarkers for predicting of pulmonary hypertension in patients with left heart disease
}

Lanlan Tan

Chongqing Medical University

Peng Jin

Army University Xinqiao Hospital

Qi Zhou

Chongqing Medical University

Xiaojing Wu (Dxiaojingwu1992@163.com)

Shenzhen University General Hospital https://orcid.org/0000-0002-0590-7627

Research article

Keywords: Pulmonary hypertension, Left heart disease, Heart failure, Biomarker, Mathematical model

Posted Date: February 25th, 2021

DOI: https://doi.org/10.21203/rs.3.rs-258871/v1

License: (a) (i) This work is licensed under a Creative Commons Attribution 4.0 International License. Read Full License 


\section{Abstract}

Background: Pulmonary hypertension $(\mathrm{PH})$ insidiously occurs in patients with left

heart disease (LHD), but its diagnosis is often delayed due to the lack of specific symptoms. This observational study aimed to identify a biomarker panel for the noninvasive detection of PH in LHD patients.

Methods: Patients with LHD were consecutively recruited and analyzed for correlations between the pulmonary artery systolic pressure (PASP) and levels of routine serum biomarkers, and 100 age-matched subjects served as healthy controls. The diagnostic accuracy of biomarkers was assessed by receiver operating characteristic (ROC) curve analysis. Forward stepwise binary logistic regression was performed to determine the optimal combination of biomarkers for predicting $\mathrm{PH}$.

Results: A total of 426 LHD patients were divided into the LHD group ( $n=216, P A S P<35 \mathrm{mmHg}$ ) and $\mathrm{PH}$ LHD group ( $n=210$, PASP $\geq 35 \mathrm{mmHg}$ ). The ranges of routinely examined biomarkers were significantly different between the two groups. The levels of biomarkers, including brain natriuretic peptide (BNP), total bilirubin (TB), direct bilirubin (DB), indirect bilirubin (IB), red cell distribution width (RDW), uric acid (UA), and cystatin c (Cys-C), were higher, while high-density lipoprotein (HDL) levels were lower in the PH-LHD group than in the LHD and healthy control groups. Positive correlations were found between the PASP and levels of BNP, Cys-C, UA, bilirubin, and RDW, while a negative correlation was found between the PASP and HDL level. A mini program based on the formula " $\mathrm{P}=1 /(1+\exp (6,314-0.898 \times[\mathrm{BNP}] / 1000-$ $0.146 \times[\mathrm{DB}]-0.318 \times[\mathrm{RDW}])$ )" was developed using forward stepwise binary regression for calculating the probability of $\mathrm{PH}$. The combination of BNP, DB, and RDW (cutoff, 0.256 ) demonstrated a better predictive value than did individual biomarkers, with a sensitivity of 0.822 and specificity of 0.783 in the prediction of $\mathrm{PH}$ in LHD patients.

Conclusion: The combination of BNP, bilirubin, and RDW shows promise as a simple method for screening LHD patients for $\mathrm{PH}$.

\section{Background}

Pulmonary hypertension (PH) is a common complication of left heart disease (LHD), and PH due to LHD (PH-LHD) has negative outcomes [1]. PH develops in LHD, but its symptoms are nonspecific. Both $\mathrm{PH}$ and LHD manifest with dyspnea, fatigue, and exercise intolerance. It is therefore difficult to identify PH in LHD patients by clinical symptoms.

$\mathrm{PH}$ is a hemodynamic condition, and invasive right heart catheterization $(\mathrm{RHC})$ has been regarded as the gold standard in its diagnosis. Currently, however, RHC is not a routine examination for LHD patients in clinical practice. Invasive hemodynamics are reported in severe or end-stage patients [2], and conventional echocardiography continues to be appropriate in most LHD patients. 
Circulating biomarkers have been proposed as potentially noninvasive and objective diagnostic and prognostic markers, as well as markers of response to therapy. In contrast to echo, biomarkers reflect a disease-associated molecular change in bodily tissues and fluids [3]. Serum biomarkers involving endothelial, inflammatory, and right ventricular (RV) dysfunction, as well as metabolic mediators, have been studied in pulmonary arterial hypertension $(\mathrm{PAH})[4,5]$. However, $\mathrm{PH}$ has a highly complex etiology. Unlike idiopathic pulmonary arterial hypertension (IPAH), which originates from a genetic or family background [6], PH-LHD usually develops in LHD in response to a passive backward transmission of filling pressures [7]. The biomarker characteristics of PH-LHD remain largely unknown. To find a reliable marker that could help to simply screen PH in LHD patients using routinely performed blood tests, in this study, we investigated the difference between routinely examined serum biomarkers in LHD patients with and without $\mathrm{PH}$ and analyzed their predictive value for $\mathrm{PH}$ in LHD patients.

\section{Methods}

\section{Patients and study design}

LDH patients of the cardiovascular department of the Second Affiliated Hospital of Chongqing Medical University and Army University Xinqiao Hospital were consecutively studied from January 2014 to July 2019. According to the guidelines on the classification of PH, eligible LHD subjects included patients with heart failure with reduced ejection fraction (HFrEF), heart failure with preserved ejection fraction (HFpEF), and cardiac valve disease $[8,9]$. The etiology of heart failure includes coronary artery disease, hypertension and cardiomyopathy, and there is no evidence for connective tissue disease, congenital heart disease, diabetes, or lung disease to be included in the etiology of heart failure. Cardiac valve disease includes diseases affecting the aortic and mitral valves. Patients in $\mathrm{PH}$ groups 1, 3, 4, and 5 were excluded. The pulmonary artery systolic pressure (PASP) was examined by echocardiography on the day of admission. We finally enrolled 426 cases with LHD. Based on the PASP, the subjects were further divided into the LHD control group (PASP $<35 \mathrm{mmHg}$ ) and the PH-LHD group (PASP $\geq 35 \mathrm{mmHg}$ ) based on the Guidelines for the Echocardiographic Assessment of the Right Heart in Adults [10]. Another 100 age- and sex-matched subjects served as healthy controls. After informed consent was obtained, peripheral venous blood was drawn from all the enrolled subjects for routine clinical examination. The study protocol conforms to the ethical guidelines of the 1975 Declaration of Helsinki and has been approved by the institutional ethical committee of the Second Affiliated Hospital of Chongqing Medical University, Xinqiao Hospital of Army University and Shenzhen University General Hospital.

\section{Clinical measurements}

The levels of uric acid (UA), creatinine, bilirubin, cystatin C (Cys-C), and lipoproteins (high-density lipoprotein [HDL] and low-density lipoprotein [LDL]) were assayed using a Beckman Coulter AU5800 system (USA). The red cell distribution width (RDW) was assayed in a routine blood analysis using a Sysmex XN-20 analyzer (Sysmex, Japan). The levels of brain natriuretic peptide (BNP) and troponin I $(T n I)$ were assayed by a ReLIA Analyzer SSJ-2 using the reagents provided by the manufacturer (ReLIA Biotechnologies, USA). 
Echocardiographic recordings were obtained in all subjects using a GE VIVID7 (GE Healthcare, Norway) color Doppler ultrasound system. Data were stored on a DVD and analyzed by two independent investigators. The simplified Bernoulli equation describes the relationship between tricuspid regurgitation velocity and the peak pressure gradient of tricuspid regurgitation, where the peak pressure gradient of tricuspid regurgitation $=4 \times$ (tricuspid regurgitation velocity). The PASP was calculated as the tricuspid regurgitation pressure gradient plus the estimated right atrial pressure. The left ventricular ejection fraction (LVEF) was calculated using the Teichholz method and the modified biplane Simpson method, as previously described [11].

Blood pressure (BP) and heart rate (HR) were measured with an Omron HEM-6200 monitor (Japan).

\section{Statistical analysis}

The statistical analysis was performed using SPSS software (IBM Corp.,version 20.0). All continuous variables are summarized as medians and interquartile ranges, and all categorical variables are expressed as proportions. Significant differences in the biomarker levels between the groups were determined using a Kruskal-Wallis nonparametric test followed by pairwise multiple comparisons. Correlations between the PASP and biomarker levels were assessed by calculating Spearman correlation coefficients. The diagnostic accuracy of serum biomarker levels was assessed by receiver operating characteristic (ROC) curve analysis. Forward stepwise binary logistic regression analyses were performed to determine the optimal combination of biomarkers for diagnosing PH-LHD. For the regression analyses, the liberal entrance criterion was 0.05 and the liberal exit criterion was $0.10 ; p<0.05$ was considered statistically significant.

\section{Results}

\section{Clinical characteristics}

The study sample comprised 100 healthy control subjects, 216 LHD control patients, and $210 \mathrm{PH}$-LHD patients. The average age, sex, and BMI were comparable between the three groups. The proportions of LHD in the patient groups were as follows: coronary artery disease (CAD, 44.91\%), hypertension (14.35\%), cardiomyopathy (21.30\%), and cardiac valve disease (19.44\%) in the LHD group; and CAD $(28.74 \%)$, hypertension (12.57\%), cardiomyopathy (27.05\%), and cardiac valve disease (31.66\%) in the PH-LHD group. The median PASP was $26.00 \mathrm{mmHg}$ (interquartile range, [IQR], 10.00 to $33.00 \mathrm{mmHg}$ ) in the healthy controls, $26.00 \mathrm{mmHg}$ (IQR, 10.00 to $34.00 \mathrm{mmHg}$ ) in the LHD control group, and $62.00 \mathrm{mmHg}$ (IQR, 55.00 to $70.25 \mathrm{mmHg}$ ) in the PH-LHD group. The pulmonary artery diameter (PAD) was $22.00 \mathrm{~mm}$ (interquartile range [IQR], 21.00 to $23.80 \mathrm{~mm}$ ) in the healthy controls, $23.00 \mathrm{~mm}$ (IQR, 22.00 to $25.00 \mathrm{~mm}$ ) in the LHD control group, and $26.00 \mathrm{~mm}$ (IQR, 24.00 to $29.00 \mathrm{~mm}$ ) in the PH-LHD group. Compared with those in the LHD control and healthy control groups, the PASP and PAD were significantly increased, the left atrium and ventricle were enlarged, and cardiac function was decreased in the PH-LHD group. Furthermore, compared with those in the LHD group, the percentage of patients with New York Heart 
Association (NYHA) disease grades III and IV was increased and the LVEF was decreased in the PH-LHD group (Table 1 and Fig. 1). 
Table 1

Basic clinical characteristics

\begin{tabular}{|c|c|c|c|c|}
\hline Variables & $\begin{array}{l}\text { Healthy } \\
\text { control } \\
(n= \\
100)\end{array}$ & $\begin{array}{l}\text { LHD control } \\
(n=216)\end{array}$ & $\begin{array}{l}\text { PH-LHD } \\
(n=210)\end{array}$ & $\begin{array}{l}P \\
\text { value }\end{array}$ \\
\hline LHD (n, \%) & & & & - \\
\hline CAD & - & $97(44.91)$ & $60(28.74)$ & - \\
\hline Cardiomyopathy & - & $46(21.30)$ & $57(27.05)$ & - \\
\hline CVD & - & $42(19.44)$ & $66(31.66)$ & - \\
\hline Hypertension & - & $31(14.35)$ & $27(12.57)$ & - \\
\hline Age (years) & $\begin{array}{l}58.50 \\
(52.00 \\
63.50)\end{array}$ & $62.00(52.00,68.75)$ & $62.00(54.50,69.00)$ & 0.088 \\
\hline Female (n, \%) & $\begin{array}{l}30 \\
(46.88)\end{array}$ & $92(42.59)$ & $63(45.99)$ & 0.747 \\
\hline Smoking (n, \%) & $\begin{array}{l}25 \\
(39.06)\end{array}$ & $91(42.13)$ & $51(37.23)$ & 0.648 \\
\hline BMI $\left(\mathrm{kg} / \mathrm{m}^{2}\right)$ & $\begin{array}{l}23.47 \\
(21.65 \\
25.85)\end{array}$ & $23.91(21.48,25.95)$ & $22.49(20.33,25.88)$ & 0.063 \\
\hline SBP (mmHg) & $\begin{array}{l}125.50 \\
(114.25, \\
135.75)\end{array}$ & $128.00(114.25,140.00)$ & $123.00(110.00,136.00) \dagger$ & 0.036 \\
\hline DBP (mmHg) & $\begin{array}{l}74.00 \\
(70.00 \\
80.00)\end{array}$ & $77.00(70.00,84.00)$ & $78.00(67.00,86.00)$ & 0.587 \\
\hline $\mathrm{mBP}(\mathrm{mmHg})$ & $\begin{array}{l}92.50 \\
(85.17 \\
96.67)\end{array}$ & $94.33(85.42,101.25)$ & $92.83(81.42,100.25)$ & 0.157 \\
\hline
\end{tabular}

Results are expressed as medians (interquartile ranges) or $\mathrm{n}$ (\%).

${ }^{\star} P<0.05,{ }^{*} P<0.01$ vs. healthy control group. $+P<0.05,+\dagger P<0.01$ vs. LHD control group.

LHD: left heart disease, CAD: coronary artery disease, CVD: cardiac valve disease, BMI: body mass index, SBP: systolic blood pressure, DBP: diastolic blood pressure, mBP: mean blood pressure, HR: heart rate, NYHA: New York Heart Association, PAD: pulmonary artery diameter, LAD: left atrial longitudinal diameter, LVD: left ventricular end-diastolic diameter, RAD: right atrial diameter, RVD: right ventricular end-diastolic diameter, PASP: pulmonary artery systolic pressure, LVEF: left ventricular ejection fraction, LVFS: left ventricular fractional shortening. 


\begin{tabular}{|c|c|c|c|c|}
\hline Variables & $\begin{array}{l}\text { Healthy } \\
\text { control } \\
(n= \\
100)\end{array}$ & $\begin{array}{l}\text { LHD control } \\
(n=216)\end{array}$ & $\begin{array}{l}\text { PH-LHD } \\
(n=210)\end{array}$ & $\begin{array}{l}P \\
\text { value }\end{array}$ \\
\hline HR (beats/min) & $\begin{array}{l}77.00 \\
(70.50 \\
86.00)\end{array}$ & $78.00(68.00,85.75)$ & $80.00(70.25,92.00)+$ & 0.017 \\
\hline NYHA grade (n, \%) & & $\star \star$ & $* *,+\dagger$ & $\begin{array}{l}<.001 \\
0.00\end{array}$ \\
\hline 1 & - & $17(7.87)$ & $2(0.95)$ & \\
\hline II & - & $104(48.15)$ & $20(9.52)$ & \\
\hline III & - & $79(36.57)$ & $118(56.19)$ & \\
\hline IV & - & $16(7.41)$ & $70(33.34)$ & \\
\hline \multicolumn{5}{|l|}{ Echocardiography } \\
\hline PAD (mm) & $\begin{array}{l}22.00 \\
(21.00 \\
23.80)\end{array}$ & $23.00(22.00,25.00) * \star$ & $26.00(24.00,29.00) * *,+†$ & $\begin{array}{l}<.001 \\
0.001\end{array}$ \\
\hline $\operatorname{LAD}(\mathrm{mm})$ & $\begin{array}{l}32.00 \\
(30.00 \\
34.00)\end{array}$ & $39.00(34.00,45.00)$ ** & $48.00(42.00,52.00) * *,+†$ & $\begin{array}{l}< \\
0.001\end{array}$ \\
\hline LVD (mm) & $\begin{array}{l}45.00 \\
(42.00 \\
47.00)\end{array}$ & $48.00(45.00,57.75)$ ** & $57.00(50.00,65.00) * *,+†$ & $\begin{array}{l}<.001 \\
0.001\end{array}$ \\
\hline $\mathrm{RAD}(\mathrm{mm})$ & $\begin{array}{l}35.00 \\
(33.00 \\
36.75)\end{array}$ & $36.00(34.00,42.00)$ * & $46.00(42.00,50.00) * \star, t+$ & $\begin{array}{l}<.001 \\
0.001\end{array}$ \\
\hline $\mathrm{RVD}(\mathrm{mm})$ & $\begin{array}{l}34.00 \\
(32.00 \\
35.00)\end{array}$ & $35.00(33.00,37.00)$ * & $42.00(37.00,46.00) * \star, t+$ & $\begin{array}{l}<.001 \\
0.001\end{array}$ \\
\hline PASP $(\mathrm{mmHg})$ & $\begin{array}{l}26.00 \\
(10.00 \\
33.00)\end{array}$ & $26.00(10.00,34.00)$ & $62.00(55.00,70.25) * *,+十$ & $\begin{array}{l}<.001 \\
0.001\end{array}$ \\
\hline
\end{tabular}

Results are expressed as medians (interquartile ranges) or $\mathrm{n}(\%)$.

${ }^{\star} P<0.05,{ }^{*} P<0.01$ vs. healthy control group. $+P<0.05,++P<0.01$ vs. LHD control group.

LHD: left heart disease, CAD: coronary artery disease, CVD: cardiac valve disease, BMI: body mass index, SBP: systolic blood pressure, DBP: diastolic blood pressure, mBP: mean blood pressure, HR: heart rate, NYHA: New York Heart Association, PAD: pulmonary artery diameter, LAD: left atrial longitudinal diameter, LVD: left ventricular end-diastolic diameter, RAD: right atrial diameter, RVD: right ventricular end-diastolic diameter, PASP: pulmonary artery systolic pressure, LVEF: left ventricular ejection fraction, LVFS: left ventricular fractional shortening. 


\begin{tabular}{|c|c|c|c|c|}
\hline Variables & $\begin{array}{l}\text { Healthy } \\
\text { control } \\
(n= \\
100)\end{array}$ & $\begin{array}{l}\text { LHD control } \\
(n=216)\end{array}$ & $\begin{array}{l}\text { PH-LHD } \\
(n=210)\end{array}$ & $\begin{array}{l}P \\
\text { value }\end{array}$ \\
\hline LVEF (\%) & $\begin{array}{l}63.00 \\
(59.00 \\
66.00)\end{array}$ & $61.00(47.00,65.00) * \star$ & $42.00(33.00,59.00) * \star,+†$ & $\begin{array}{l}<.001 \\
0.01\end{array}$ \\
\hline LVFS (\%) & $\begin{array}{l}33.50 \\
(31.25 \\
36.00)\end{array}$ & $32.00(23.55,35.38) * \star$ & $21.00(16.00,31.00) * \star$, †† & $\begin{array}{l}<.001 \\
0.01\end{array}$ \\
\hline Treatments & - & $\begin{array}{l}\text { Beta-blockers }(66.20 \%), \\
\text { ACEl/ARB (71.76\%), } \\
\text { aldosterone antagonist } \\
(43.98 \%), \text { diuretics } \\
(47.69 \%), \text { statins } \\
(67.59 \%), \text { digitalis } \\
(14.35 \%), \text { beraprost } \\
(7.41 \%), \text { anticoagulant } \\
\text { and/or antiplatelet } \\
\text { therapy }(79.17 \%)\end{array}$ & $\begin{array}{l}\text { Beta-blockers }(68.61 \%), \\
\text { ACEI/ARB }(81.75 \%) \text {, } \\
\text { aldosterone antagonist } \\
\text { (78.83\%), diuretics } \\
(86.86 \%), \text { statins }(42.34 \%) \text {, } \\
\text { digitalis }(53.28 \%), \\
\text { beraprost }(31.39 \%), \\
\text { anticoagulant and/or } \\
\text { antiplatelet therapy } \\
\text { (70.07\%) }\end{array}$ & - \\
\hline \multicolumn{5}{|c|}{ Results are expressed as medians (interquartile ranges) or $n(\%)$. } \\
\hline \multicolumn{5}{|c|}{${ }^{\star} P<0.05, * \star P<0.01$ vs. healthy control group. $\dagger P<0.05,+\dagger P<0.01$ vs. LHD control group. } \\
\hline \multicolumn{5}{|c|}{$\begin{array}{l}\text { LHD: left heart disease, CAD: coronary artery disease, CVD: cardiac valve disease, BMI: body mass } \\
\text { index, SBP: systolic blood pressure, DBP: diastolic blood pressure, mBP: mean blood pressure, HR: } \\
\text { heart rate, NYHA: New York Heart Association, PAD: pulmonary artery diameter, LAD: left atrial } \\
\text { longitudinal diameter, LVD: left ventricular end-diastolic diameter, RAD: right atrial diameter, RVD: right } \\
\text { ventricular end-diastolic diameter, PASP: pulmonary artery systolic pressure, LVEF: left ventricular } \\
\text { ejection fraction, LVFS: left ventricular fractional shortening. }\end{array}$} \\
\hline
\end{tabular}

\section{Biomarker levels}

The levels of biomarkers were significantly different between the LHD control and PH-LHD groups. Levels of biomarkers, including BNP, total bilirubin (TB), direct bilirubin (DB), indirect bilirubin (IB), RDW, UA, and Cys-C, were significantly higher in the PH-LHD group than in the LHD group. The level of HDL cholesterol was significantly lower in the PH-LHD group than in the LHD group. No differences were found in the TnI levels between the three groups (Table 2). 
Table 2

Levels of serum biomarkers and correlations with PASP

\begin{tabular}{|c|c|c|c|c|}
\hline Biomarkers & $\begin{array}{l}\text { Healthy control } \\
(n=100)\end{array}$ & $\begin{array}{l}\text { LHD control } \\
(n=216)\end{array}$ & $\begin{array}{l}\text { PH-LHD } \\
(n=210)\end{array}$ & $\begin{array}{l}P \\
\text { value }\end{array}$ \\
\hline $\begin{array}{l}\text { BNP } \\
(\mathrm{pg} / \mathrm{mL})\end{array}$ & $22.35(8.18,52.38)$ & $\begin{array}{l}124.00(30.30 \\
398.00) * \star\end{array}$ & $\begin{array}{l}742.00(348.00,1470.00) \\
\star \star \\
*+\dagger\end{array}$ & $\begin{array}{l}< \\
0.001\end{array}$ \\
\hline TB ( $\mu \mathrm{mol} / \mathrm{L})$ & $10.45(8.75,14.28)$ & $13.20(10.00,17.35)$ * & $21.40(15.15,33.95) * \star$,十† & $\begin{array}{l}< \\
0.001\end{array}$ \\
\hline $\begin{array}{l}\text { DB } \\
(\mu \mathrm{mol} / \mathrm{L})\end{array}$ & $1.95(1.40,2.50)$ & $2.50(1.80,3.30) * \star$ & $5.30(3.05,10.00) * *,+†$ & $<.001$ \\
\hline IB $(\mu \mathrm{mol} / \mathrm{L})$ & $8.35(7.00,12.00)$ & $10.30(8.10,13.70)$ * & $15.40(11.75,23.25) * \star,+†$ & $<.001$ \\
\hline RDW (\%) & $13.05(12.60,13.70)$ & $13.40(12.70,14.00)$ & $14.50(13.50,16.10) * \star$,†† & ¿ \\
\hline $\mathrm{UA}(\mu \mathrm{mol} / \mathrm{L})$ & $\begin{array}{l}321.00(268.25 \\
412.25)\end{array}$ & $\begin{array}{l}349.50(280.00 \\
421.25)\end{array}$ & $\begin{array}{l}481.50(359.00,585.75) \\
* *,+\dagger\end{array}$ & $<.001$ \\
\hline $\begin{array}{l}\text { Cys-C } \\
(\mathrm{mg} / \mathrm{L})\end{array}$ & $0.88(0.80,1.02)$ & $1.00(0.89,1.17)$ * & $1.19(1.01,1.52) * \star$,†† & $\begin{array}{l}< \\
0.001\end{array}$ \\
\hline $\begin{array}{l}\mathrm{HDL} \\
(\mathrm{mmol} / \mathrm{L})\end{array}$ & $1.06(0.92,1.22)$ & $1.00(0.83,1.21)$ & $0.86(0.71,1.05) * \star$,†十 & $<.001$ \\
\hline $\mathrm{Tnl}(\mathrm{ng} / \mathrm{mL})$ & $0.05(0.05,0.05)$ & $0.05(0.05,0.05)$ & $0.05(0.05,0.05)$ & 0.792 \\
\hline \multicolumn{5}{|c|}{ Results are expressed as medians (interquartile ranges). } \\
\hline \multicolumn{5}{|c|}{$\begin{array}{l}\text { PASP: pulmonary artery systolic pressure, BNP: brain natriuretic peptide, TB: total bilirubin, DB: direct } \\
\text { bilirubin, IB: indirect bilirubin, RDW: red cell distribution width, UA: uric acid, Cys-C: cystatin c, HDL: } \\
\text { high-density lipoprotein, Tnl: troponin I. }\end{array}$} \\
\hline
\end{tabular}

\section{Correlation between biomarkers and PASP}

The levels of the biomarkers BNP, TB, DB, IB, RDW, UA, and Cys-C, but not HDL, were positively correlated with the PASP. No correlation was found between Tnl and PASP (Fig. 2).

\section{ROC curve analysis}

The diagnostic accuracies of each biochemistry marker determined by the ROC curve analysis are shown in Table 3. The combination of BNP, DB, and RDW demonstrated a better predictive value than the individual biomarkers, with an area under the curve (AUC) of 0.858 and Youden index of 0.605. Using the forward stepwise binary logistic regression model, a mini program based on the formula $P=1 /(1+$ $\exp (6,314-0.898 \times[\mathrm{BNP}] / 1000-0.146 \times[\mathrm{DB}]-0.318 \times[\mathrm{RDW}]))$ was developed to calculate the predicted probability of $\mathrm{PH}$. The combination of BNP, DB, and RDW (cutoff, 0.256 ) had a sensitivity of 0.822 and specificity of 0.783 in the prediction of PH in LHD patients (Fig. 3, Table 3). 
Table 3

Diagnostic accuracy of serum biomarkers for PH in LHD patients

\begin{tabular}{|c|c|c|c|c|c|c|c|c|}
\hline Biomarkers & AUC & SE & $95 \% \mathrm{Cl}$ & $\begin{array}{l}P \\
\text { value }\end{array}$ & Cut-off & Sensitivity & Specificity & $\begin{array}{l}\text { Youden } \\
\text { index }\end{array}$ \\
\hline BNP (pg/mL) & 0.840 & 0.020 & $\begin{array}{l}0.801 \sim \\
0.879\end{array}$ & ${ }_{0.001}^{<}$ & 200.00 & 0.872 & 0.670 & 0.572 \\
\hline TB $(\mu \mathrm{mol} / \mathrm{L})$ & 0.770 & 0.026 & $\begin{array}{l}0.719 \sim \\
0.821\end{array}$ & $\begin{array}{l}< \\
0.001\end{array}$ & $\begin{array}{l}> \\
18.00\end{array}$ & 0.647 & 0.810 & 0.457 \\
\hline $\mathrm{DB}(\mu \mathrm{mol} / \mathrm{L})$ & 0.805 & 0.025 & $\begin{array}{l}0.757 \sim \\
0.853\end{array}$ & $\begin{array}{l}< \\
0.001\end{array}$ & $>3.40$ & 0.714 & 0.817 & 0.531 \\
\hline IB $(\mu \mathrm{mol} / \mathrm{L})$ & 0.743 & 0.027 & $\begin{array}{l}0.690 \sim \\
0.796\end{array}$ & $<.001$ & 72.80 & 0.692 & 0.733 & 0.425 \\
\hline RDW (\%) & 0.768 & 0.025 & $\begin{array}{l}0.720 \sim \\
0.816\end{array}$ & $\begin{array}{l}< \\
0.001\end{array}$ & $\begin{array}{l}> \\
13.70\end{array}$ & 0.689 & 0.717 & 0.406 \\
\hline $\mathrm{UA}(\mu \mathrm{mol} / \mathrm{L})$ & 0.733 & 0.027 & $\begin{array}{l}0.679 \sim \\
0.787\end{array}$ & $\begin{array}{l}< \\
0.001\end{array}$ & $\begin{array}{l}> \\
454.00\end{array}$ & 0.559 & 0.862 & 0.421 \\
\hline Cys-C (mg/L) & 0.708 & 0.028 & $\begin{array}{l}0.654 \sim \\
0.763\end{array}$ & $<.001$ & $>1.06$ & 0.721 & 0.658 & 0.379 \\
\hline $\begin{array}{l}\text { HDL } \\
\text { (mmol/L) }\end{array}$ & 0.670 & 0.029 & $\begin{array}{l}0.614 \sim \\
0.726\end{array}$ & $\begin{array}{l}< \\
0.001\end{array}$ & $<1.20$ & 0.729 & 0.526 & 0.255 \\
\hline $\begin{array}{l}\text { BNP, DB \& } \\
\text { RDW (PP) }\end{array}$ & 0.858 & 0.020 & $\begin{array}{l}0.819 \sim \\
0.897\end{array}$ & $<.001$ & $\begin{array}{l}> \\
0.256\end{array}$ & 0.822 & 0.783 & 0.605 \\
\hline \multicolumn{9}{|c|}{$\begin{array}{l}\text { AUC: area under the curve, SE: standard error, Cl: confidence interval, BNP: brain natriuretic peptide, } \\
\text { TB: total bilirubin, DB: direct bilirubin, IB: indirect bilirubin, RDW: red cell distribution width, UA: uric } \\
\text { acid, Cys-C: cystatin c, HDL: high-density lipoprotein. PP: predicted probability according to prediction } \\
\text { model generated using logistic regression analysis. }\end{array}$} \\
\hline
\end{tabular}

\section{Discussion}

The potential of biomarker combinations is currently of considerable interest in the diagnosis of $\mathrm{PH}$. Our study showed that the levels of routinely examined serum biomarkers were significantly different between the LHD control and PH-LHD patients. The correlation analysis showed positive correlations between the PASP and levels of BNP, Cys-C, UA, bilirubin, and RDW, while a negative correlation was found between PASP and HDL. The combination of BNP, bilirubin, and RDW was found to exhibit the best diagnostic accuracy for $\mathrm{PH}$ in LHD patients.

LHD involving HFpEF, HFrEF, and heart valves is the most frequent cause of $\mathrm{PH}$. $\mathrm{PH}$-LHD belongs to group $2 \mathrm{PH}$, with the following hemodynamic characteristics: mean pulmonary arterial pressure (mPAP) $>20$ $\mathrm{mmHg}$ and pulmonary capillary wedge pressure (PCWP) $>15 \mathrm{mmHg}[8,9]$. PH insidiously occurs in LHD, and its diagnosis is often delayed due to the lack of specific symptoms. $\mathrm{RHC}$ is the gold standard in the diagnosis of $\mathrm{PH}$, but it is often declined by LHD patients due to its invasive nature. Transthoracic 
echocardiography remains a widely available tool in cardiology clinics worldwide. In our study, we consecutively enrolled 426 LHD patients and divided them into the LHD group and the PH-LHD group. With regard to etiology, we found that the ratio between cardiomyopathy and valve disease prevalence was higher than that between CAD and hypertension prevalence in the PH-LHD group. However, the ratio between $C A D$ and hypertension prevalence was higher than that between cardiomyopathy and valve disease prevalence in the LHD control group. Similar to previous reports [7, 12], our study showed that compared with that in the LHD control group, cardiac function, assessed by the NYHA grade and LVEF, was significantly decreased in the PH-LHD group. Unlike LHD control patients, patients with PH-LHD often exhibited enlargement and remodeling of the right atrium and right ventricle. Our study indicated that patients with LHD due to cardiomyopathy and valve disease, especially those with severe cardiac function damage, are prone to $\mathrm{PH}$.

Circulating biomarkers have been investigated as potentially noninvasive and objective measures for the diagnosis and prognosis of diseases in daily clinical practice. In our study, we found that the levels of routinely examined biomarkers were significantly different between the PH-LHD and LHD control groups. The levels of BNP, TB, DB, IB, RDW, UA, and Cys-C were significantly higher and the HDL level was lower in the PH-LHD group than in the LHD group.

BNP belongs to the natriuretic peptide family and has potent vasodilator, hypertrophic, and proinflammatory properties. Increasing evidence has shown that circulating levels of BNP are correlated with the mPAP and pulmonary vascular resistance (PVR) in patients with PAH $[13,14]$. Our study showed that BNP was positively correlated with PASP. BNP exerts myriad cardiovascular effects [15]. The sensitivity of BNP alone (cutoff, $200.00 \mathrm{pg} / \mathrm{mL}$ ) in predicting PH was high (0.874), but its specificity was low (0.630).

Patients with right heart failure often exhibit elevated bilirubin levels because of congestion and low perfusion. Takeda et al. [16] enrolled 37 patients with PAH and followed them for a median of 635 days. They found that elevated serum bilirubin was a risk factor for death. In our study, the levels of IB and DB were significantly higher in the PH-LHD group than in the LHD control group. Both IB and DB were positively correlated with PASP.

UA is the metabolite of purine nucleotide, and an elevated UA level represents a higher activity of oxidative metabolism. Recent evidence suggests that UA inhibits acetylcholine-mediated vasodilation by acting on the vascular endothelium [17]. In isolated porcine pulmonary artery segments, UA reduced nitric oxide levels in pulmonary arterial endothelial cells and inhibited acetylcholine-induced vasodilation [18]. Previous studies have shown that serum UA levels are associated with the severity of IPAH and ventricular dysfunction $[19,20]$. Cys-C, a novel marker of renal function, predicts left heart failure and cardiovascular mortality. Fenster et al. [21] found that the Cys-C level accurately correlates with RV pressure, function, and morphology. In our study, although the levels of UA and Cys-C were within normal ranges in both the LHD control and PH-LHD groups, the levels were significantly different between the 
groups. Serum levels of UA and Cys-C were significantly higher in the PH-LHD group than in the LHD group. Additionally, both UA and Cys-C were positively correlated with PASP.

The HDL level is a strong, independent, inverse predictor of cardiovascular disease, and it is not influenced by statins or diet. Previous studies by Zhao et al. [22] and Heresi et al. [23] showed that serum HDL cholesterol levels were significantly lower in patients with IPAH than in control individuals, and the levels were decreased in proportion to the severity of the World Health Organization functional class. In our study, the HDL level in the PH-LHD group was significantly lower than that in the LHD control group and was negatively correlated with the PASP. HDL alone demonstrated a sensitivity of 0.729 and specificity of 0.526 in predicting PH in LHD patients.

The RDW, which is measured as part of the full blood count in standard hospital analyses and quantifies the variability in red blood cell size, had been shown to be valuable for assessing survival and prognosis in cardiovascular disease patients. A study by Rhodes et al. [24] demonstrated that the RDW outperforms the BNP level in predicting survival in IPAH patients. In our study, the RDW was significantly higher in the PH-LHD group than in the LHD control group and was positively correlated with the PASP. The RDW alone demonstrated a sensitivity of 0.690 and specificity of 0.689 in predicting PH in LHD patients.

The ideal serum biomarker for use in $\mathrm{PH}$ remains elusive despite recent extensive work. A diseasespecific, clinically useful, and noninvasive biomarker for $\mathrm{PH}$ does not exist, partly due to the heterogeneity and complex etiology of $\mathrm{PH}$. Our study showed that the ranges of routinely examined biomarkers, including BNP, bilirubin, UA, Cys-C, and RDW, although not specific, were significantly different. Using a bivariate logistic regression model, we found that the combination of BNP, DB, and RDW demonstrated a higher AUC and Youden index, and both the sensitivity and specificity increased relative to those of individual biomarkers. Our study indicated that a multiple biomarker approach is helpful for the screening and identification of PH in LHD patients. Moreover, using the formula generated by the bivariate logistic regression analysis, we developed a mini program to calculate the predicted probability of $\mathrm{PH}$ based on BNP, DB and RDW, which might be a simple tool to help recognize and screen PH in LHD patients in clinical practice.

There are several limitations to this study. One limitation is the inaccuracy of the noninvasive methods in assessing pulmonary artery pressure. However, although more accurate, invasive RHC measurements cannot be obtained in healthy and LHD controls due to ethical considerations. Noninvasive echocardiography is widely used in clinical practice. Moreover, according to the guidelines for $\mathrm{PH}$ diagnosis, the diagnostic accuracy of $\mathrm{PH}$ by echocardiography is close to that by $\mathrm{RHC}$, especially when PASP $>50 \mathrm{mmHg}$. The second limitation is the limited sample number. An expanded random sample across all genders and ages may be more representative. We also need a large cohort to verify the prediction value for PH-LHD using the mathematical model.

\section{Conclusion}


Collectively, the levels of routinely examined biomarkers were significantly different between LHD control and PH-LHD patients, and changes in the levels of these biomarkers may predict the severity of $\mathrm{PH}$ in patients with LHD. The combination of BNP, bilirubin, and RDW shows promise as a simple screening tool for $\mathrm{PH}$ in LHD patients.

\section{Abbreviations}

BNP: Brain natriuretic peptide; DB: Direct bilirubin; HFpEF: Heart failure with preserved ejection fraction; HFrEF: Heart failure with reduced ejection fraction; IQR: Interquartile range; LHD: Left heart disease; LVEF: Left ventricular ejection fraction; mPAP: Mean pulmonary arterial pressure; PAH: Pulmonary arterial hypertension; PASP: Pulmonary artery systolic pressure; PH: Pulmonary hypertension; RDW: Red cell distribution width; RHC: Right heart catheterization; ROC: Receiver operating characteristic

\section{Declarations}

\section{Ethics approval and consent to participate}

The study protocol conforms to the ethical guidelines of the 1975 Declaration of Helsinki and was approved by the institutional ethical committees of Chongqing Medical University Second Affiliated Hospital, Xinqiao Hospital of Army University and Shenzhen University General Hospital.

\section{Consent for publication}

Not applicable.

\section{Availability of data and materials}

The datasets generated and/or analyzed during the current study are not publicly available due to privacy or ethical restrictions, but are available from the corresponding author on reasonable request.

\section{Competing interests}

The authors declare that there are no conflicts of interest.

\section{Funding}

This study was supported by grants from the National Scientific Foundation of China (NSFC, 81270109), the Natural Scientific Foundation of Guangdong (2019A1515011421), the Natural Scientific Foundation of Shenzhen (JCYJ20190808122207499) and Shenzhen University General Hospital (SUGH2018QD048). The funders had no role in the study design, the collection, analysis and interpretation of the data, writing of the manuscript, or in the decision to submit the article for publication.

\section{Authors' Contributions}


LT and PJ contributed equally to this work and share the first authorship. QZ and XW contributed equally to this work and share the corresponding authorship. QZ and XW contributed to the study concept and design. Material preparation and data collection and analysis were performed by PJ and LT. The draft of the manuscript was written by XW. All authors read and approved the final manuscript.

\section{Acknowledgments}

We would like to thank all the participants for their support.

\section{References}

1 Weitsman T, Weisz G, Farkash R et al. Pulmonary hypertension with left heart disease: prevalence, temporal shifts in etiologies and outcome. Am J Med, 2017,130:1272-79.

2 D'Alto M, Bossone E, Opotowsky AR, Ghio S, Rudski LG, Naeije R.Strengths and weaknesses of echocardiography for the diagnosis of pulmonary hypertension. Int J Cardiol, 2018, 263:177-183.

3 Jardim C, Souza R. Biomarkers and prognostic indicators in pulmonary arterial hypertension. Curr Hypertens Rep, 2015, 17:556.

4 Pezzuto B, Badagliacca R, Poscia R et al. Circulating biomarkers in pulmonary arterial hypertension: update and future direction. J Heart Lung Transplant, 2015,34:282-305.

5 Cracowski JL, Leuchte HH. The potential of biomarkers in pulmonary arterial hypertension. Am J Cardiol, 2012,110:32S-8S.

6 Machado RD, Southgate L, Eichstaedt CA et al. Pulmonary arterial hypertension: a current perspective on established and emerging molecular genetic defects. Hum Mutat, 2015,36:1113-27.

7 Gerges M, Gerges C, Pistritto AM et al. Pulmonary hypertension in heart failure. Epidemiology, right ventricular function, and survival. Am J Respir Crit Care Med, 2015,192:1234-46.

8 Vachiéry JL, Tedford RJ, Rosenkranz S et al. Pulmonary hypertension due to left heart disease. Eur Respir J, 2019,53:1801897.

9 Guazzi M, Ghio S, Adir Y. Pulmonary Hypertension in HFpEF and HFrEF: JACC Review Topic of the Week. J Am Coll Cardiol, 2020, 76:1102-1111.

10 Rudski LG, Lai WW, Afilalo J et al. Guidelines for the echocardiographic assessment of the right heart in adults: a report from the American Society of Echocardiography endorsed by the European Association of Echocardiography, a registered branch of the European Society of Cardiology, and the Canadian Society of Echocardiography. J Am Soc Echocardiogr, 2010,23:685-713. 
11 Allemann Y, Stuber T, de Marchi SF et al. Pulmonary artery pressure and cardiac function in children and adolescents after rapid ascent to 3,450 m. Am J Physiol Heart Circ Physiol, 2012,302:H2646-53.

12 Rosenkranz S, Gibbs JS, Wachter R, De Marco T, Vonk-Noordegraaf A, Vachiéry JL. Left ventricular heart failure and pulmonary hypertensiondagger. Eur Heart J, 2016,37:942-54.

13 Rosenthal JL, Jacob MS. Biomarkers in pulmonary arterial hypertension. Curr Heart Fail Rep, 2014, 11:477-84.

14 Casserly B, Klinger JR. Brain natriuretic peptide in pulmonary arterial hypertension: biomarker and potential therapeutic agent. Drug Des Devel Ther, 2009, 3:269-87.

15 Mahadavan G, Nguyen TH, Horowitz JD. Brain natriuretic peptide: a biomarker for all cardiac disease? Curr Opin Cardiol, 2014,29:160-6.

16 Takeda Y, Takeda Y, Tomimoto S, Tani T, Narita H, Kimura G. Bilirubin as a prognostic marker in patients with pulmonary arterial hypertension. BMC Pulm Med, 2010,10:22.

17 Khosla UM, Zharikov S, Finch JL et al. Hyperuricemia induces endothelial dysfunction. Kidney Int, 2005,67:1739-42.

18 Zharikov S, Krotova K, Hu H et al. Uric acid decreases NO production and increases arginase activity in cultured pulmonary artery endothelial cells. Am J Physiol Cell Physiol, 2008,295:C1183-90.

19 Zharikov SI, Swenson ER, Lanaspa M, Block ER, Patel JM, Johnson RJ. Could uric acid be a modifiable risk factor in subjects with pulmonary hypertension? Med Hypotheses, 2010,74:1069-74.

20 Zhang CY, Ma LL, Wang LX. Relationship between serum uric acid levels and ventricular function in patients with idiopathic pulmonary hypertension. Exp Clin Cardiol, 2013,18:e37-9.

21 Fenster BE, Lasalvia L, Schroeder JD et al. Cystatin C: a potential biomarker for pulmonary arterial hypertension. Respirology, 2014,19:583-9.

22 Zhao QH, Peng FH, Wei $\mathrm{H}$ et al. Serum high-density lipoprotein cholesterol levels as a prognostic indicator in patients with idiopathic pulmonary arterial hypertension. Am J Cardiol, 2012, 110:433-9.

23 Heresi GA, Aytekin M, Newman J, DiDonato J, Dweik RA. Plasma levels of high-density lipoprotein cholesterol and outcomes in pulmonary arterial hypertension. Am J Respir Crit Care Med, 2010,182:661-8.

24 Rhodes CJ, Wharton J, Howard LS, Gibbs JS, Wilkins MR. Red cell distribution width outperforms other potential circulating biomarkers in predicting survival in idiopathic pulmonary arterial hypertension. Heart, 2011,97:1054-60.

\section{Figures}



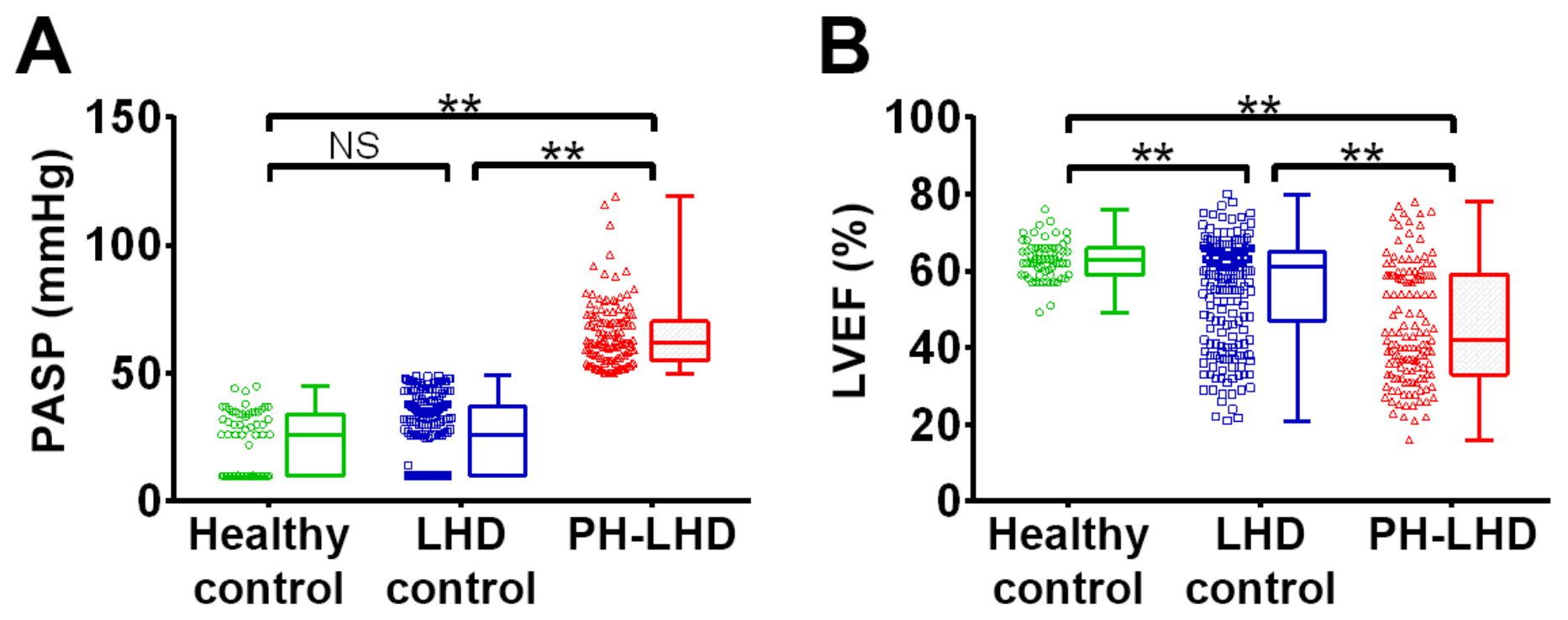

Figure 1

Distribution of the pulmonary artery systolic pressure (PASP) and left ventricular ejection fraction (LVEF) in the healthy control, LHD control, and PH-LHD groups. A: PASP; B: LVEF. 

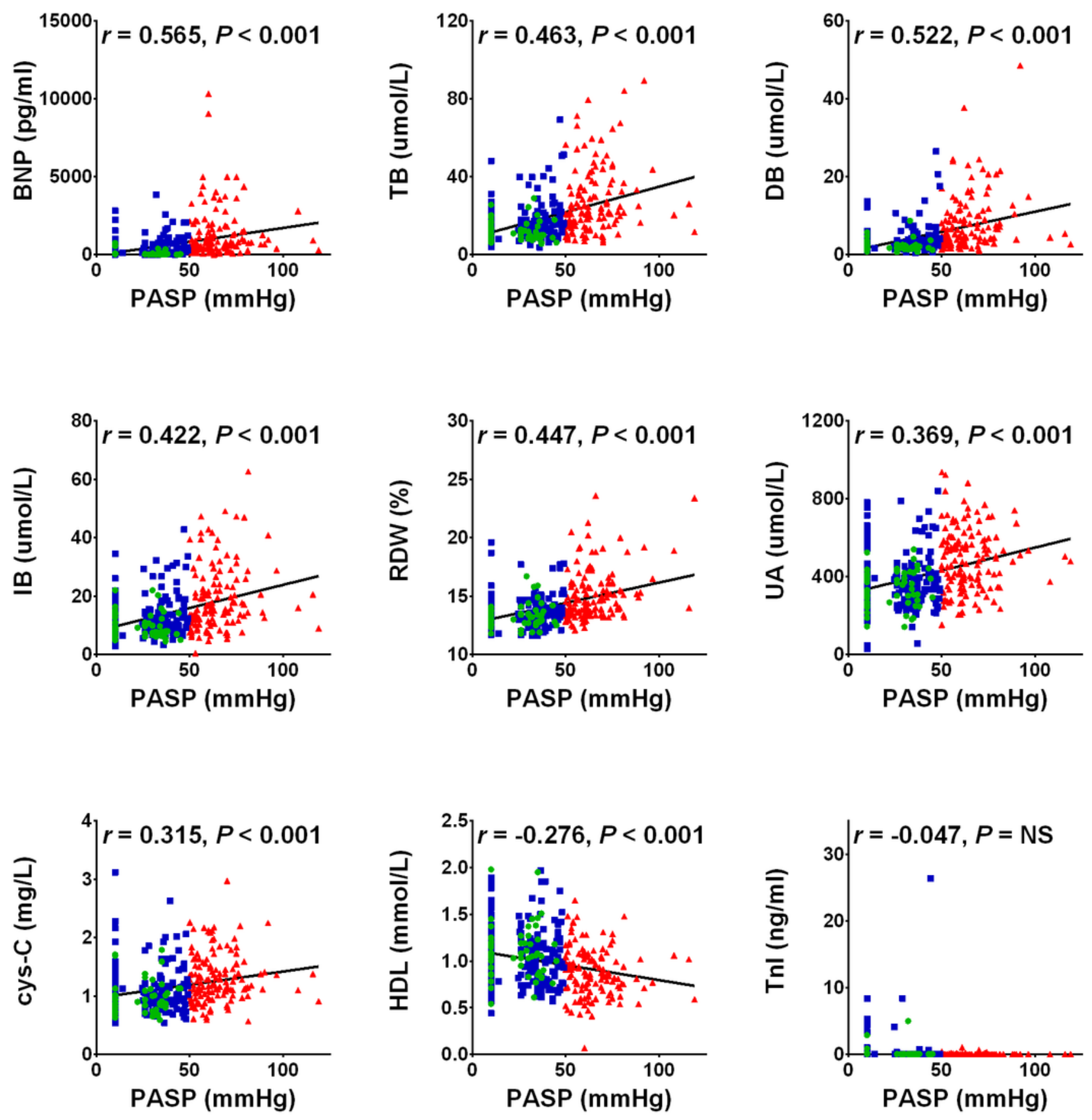

Healthy control

LHD control

$\triangle$ PH-LHD

Figure 2

Correlations between the levels of biomarkers and pulmonary artery systolic pressure (PASP). BNP = brain natriuretic peptide; Cys-C = cystatin c; DB = direct bilirubin; $\mathrm{HDL}=$ high-density lipoprotein; $\mathrm{IB}=$ indirect bilirubin; RDW = red cell distribution width; TB = total bilirubin; $\mathrm{TnI}$ = troponin I; UA: uric acid. 

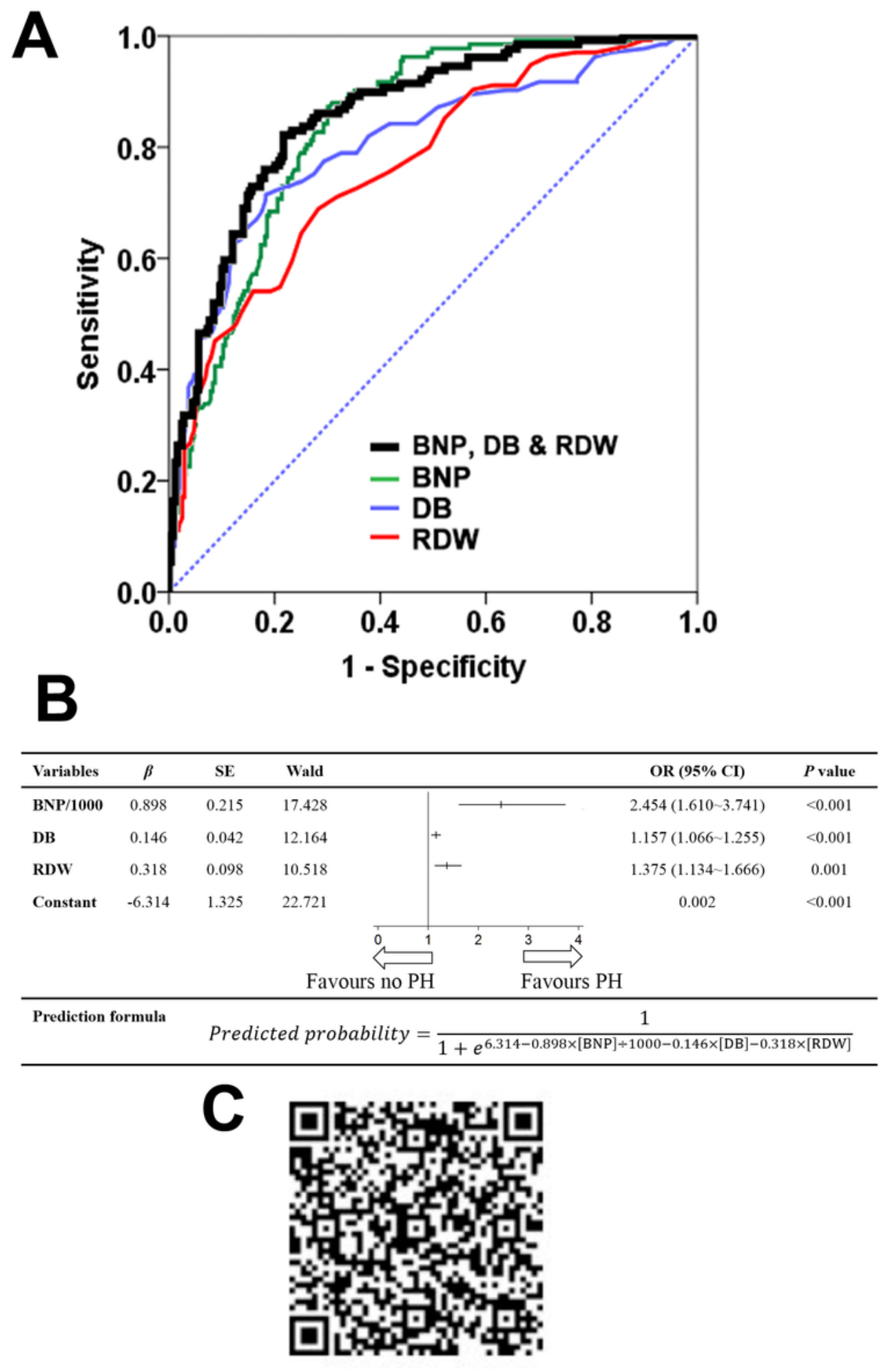

Figure 3

Combination of serum biomarkers used to predict PH in LHD patients. A: ROC curves of predicted probability obtained by the prediction formula based on brain natriuretic peptide (BNP), direct bilirubin (DB), and red cell distribution width (RDW). B: Prediction model generated using multiple logistic regression analysis. $\mathrm{C}$ : The mini program for the $\mathrm{PH}$ calculator. $\mathrm{Cl}=$ confidence interval; $\mathrm{OR}=$ odds ratio; $\mathrm{SE}=$ standard error. 